\title{
Risk Analysis of the River Bank Washout and Flooding of the Areas
}

\section{David Kereselidze, Vazha Trapaidze, Giorgi Bregvadze, Irakli Megrelidze}

Department of Hydrometeorology, Faculty of Exact \& Natural Sciences, Ivane Javakhishvili Tbilisi State University, Tbilisi, Georgia

\section{Email address:}

david.kereselidze@tsu.ge (D. Kereselidze), vazha.trapaidze@tsu.ge (V. Trapaidze), giorgi.bregvadze@tsu.ge (G. Bregvadze), iramegrelidze@gmail.com (I. Megrelidze)

\section{To cite this article:}

David Kereselidze, VazhaTrapaidze, Giorgi Bregvadze, Irakli Megrelidze. Risk Analysis of the River Bank Washout and Flooding of the Areas. Earth Sciences. Special Issue: Modern Problems of Geography and Anthropology. Vol. 4, No. 5-1, 2015, pp. 113-119.

doi: $10.11648 /$ j.earth.s.2015040501.31

\begin{abstract}
Quantitative assessment and forecasting of one or another hydrological phenomenon is important for estimation of vulnerability of natural riverside. Mechanism of riverside destruction by water is considered in the represented work as random process, which is depended both on influence of flow speed and on riverside resistance. As the indicator of this process against such influence is taken riverside characteristic - vulnerability, for determination of which is used a well-known model of the theory of reliability, called "load-strength" model. Proceeding from this fact a result obtained via theoretical formalization in the form of represented formula is considered at this stage as approximation and time factor should be taken into account in the modeling process that will be a step forward in relation to current reality.
\end{abstract}

Keywords: Riverside, Vulnerability, Probabilistic Assessment

\section{Introduction}

Modern world is in the period of unprecedented global changes. Areas of vital importance (atmosphere, hydrosphere, lithosphere and biosphere), which are necessary for human's existence are changing in various directions that can probably create a danger for well-being of both present and future generations.

According to UN experts data, against the background of established tendencies of climate changes of the last period among natural disasters, which cause certain ecological problems and social-economic difficulties, such phenomena became rather more frequently and don't give the pas, as floods and freshets.

There is also much tension around mentioned problem in Georgia, where up to 26000 big and small rivers are regitered against the diverse orographic background, and most of them fall at the western Georgia.

Rivers very often damage coastal strips of river bed, and water mass, which overflows the banks creates serious dager for settlements, industry, agriculture, communications etc.

From the ancient times the mankind had fought against floods and negative effects caused by them and had used diferent approaches and methods for it. First stages of this fight according to chronology were based on descriptive and empirical studies and they lacked the opportunities of deep scientific solution. The latter became partially implementable only after that hydrology has transformed into science, which is able to quantitatively assess one or another phenomenon and to carry out its forecasting.

Despite the fact that at present we have data on lots of fundamental scientific studies related to forecasting of floods and freshets, nevertheless we can't assert that the issue is solved at desirable level, even results of verification of already performed best works in some cases are considerably deviated from reality.

Certain progress in use of modern machinery of random processes and field theories is mentioned recently in regard to hydrological forecasting.

The practice shows that water overtopping from water-bearing artery (river bed) of the river in most cases is related to damage of coastal strip by the flow and its malfunction as of water-retaining object. Remarkable example of this fact is well-known freshet at Rioni river on January 31, 1987, when intensive lateral erosion processes caused by powerful flow originated in river bed, washed off and knocked out of action coast-protection structures (dikes) almost in twenty places, as a result of which 2-meter high wave flooded human settlements, arable and crop areas, 
communication road network, a lot of houses has been destroyed, plenty of cattle was drowned and even human losses were registered [1,2].

The foregoing and other similar examples insistently require preliminary assessment of vulnerability of riverside.

\section{Research Method}

\subsection{Determination of Probable Value of Vulnerability}

By vulnerability is meant object feature, which reflects its ability to offer resistance to current load (influence) on it, i.e. it is a feature, inverse to resistance. Degree of vulnerability is depended both on intensity and duration of loading and on existing interrelation between component elements of the object. Critical limit of vulnerability determines the range of trouble-free performance of the object. It is desirable to assess vulnerability according to all the indicators, which characterize its critical value, however it is unrealizable due to restriction of precise analytical modeling of current processes. That's why we must select one basic determining (integral) factor among characteristic indicators of critical state, according to which will be implemented the analysis of vulnerable state of the object [3].

After selection of determining factor it is possible to use well known procedures of establishment of reliability for assessment of vulnerability. Model "load-strength" is frequently used for this purpose in the theory of reliability.

In our case a bottom velocity of water flow $\left(v_{b}\right)$ can be considered as determining factor in regard to "load". Determination of approximate values of bottom velocity for any cross-section can be carried out by taking into account the values of morphometrical characteristics, hydrological and hydraulic elements of river bed, precise definition of which in case of necessity will be implemented according to direct measurements, while in regard to riverside as determining factor will be used integral characteristic of soil compaction, which determines permissible (non-washing out) speed of water flow $\left(v_{p}\right)$. The latter is the maximal value of water flow, which doesn't cause washing-out of given soil.

Let's consider a short, straight-line stretch of river bed, which can be taken as prizmatic one with uniform riverside soils. Under this assumption we may be able to formalize at some level the problem of determination of riverside vulnerability. Let's assume that in certain time period (decade) river movement at the stretch under review is steady and bottom velocity $\left(v_{b}\right)$ experiences relatively insignificant changes. Under these conditions bottom velocity of the flow can be taken as random value $v_{b}$ distribution density of which is $f_{1}\left(v_{b}\right)$, mathematical expectation is $m_{v_{b}}$ and mean square deviation is $\delta_{v_{b}}$.

In the same way we consider as random values a permissible speed of water flow $v_{p}$, distribution density of which is $f_{2}\left(v_{p}\right)$, mathematical expectation is $m_{v_{p}}$ and mean square deviation is $\delta_{v_{p}}$. It is natural that if $v_{b}$ will exceed $v_{p}$, riverside washing-off will take place, i.e. mutual consideration of random values $v_{b}$ and $v_{p}$ as of system gives us an opportunity of forecasting of riverside vulnerability. With this end we input new random value $-Z$, which is related to our start random values via general functional dependence:

$$
Z=\phi\left(V_{b}-V_{p}\right)
$$

Let's suppose as already known the probability density $f\left(V_{b}-V_{p}\right)$ of the system of random values $\left(V_{b}-V_{p}\right)$ and determine the distribution law for value $Z$. Therefore the problem of forecasting of vulnerability is considerably simplified, since we are moving from the system of two random values to one random value $\mathrm{Z}$.

As far as the dependence (1) represents certain surface in $V_{b} 0 V_{p} Z$ system, distribution function $\mathrm{G}(\mathrm{z})$ of random value $\mathrm{Z}$ will be written as follows $[4,5]$

$$
G(z)=P(Z<z)=P\left(\varphi\left(V_{b,} V_{p}\right)<z\right)
$$

where $\mathrm{z}$ is a distance from cutting plane $\mathrm{H}$ to coordinate plane $V_{b} 0 V_{p}$, which is drawn in parallel to it secant line $\mathrm{K}$, points of which satisfy the equality $\varphi\left(v_{b}, v_{p}\right)=Z$, during project onto the coordinate plane $v_{b} 0 v_{p}$ will divide the latter into two areas, and points of one of them (let us denote it as D) satisfy the condition $\varphi\left(v_{b}, v_{p}\right)<Z$. Then for fulfillment of (2) a random point $\left(V_{b}, V_{p}\right)$ must get into D area, proceeding from this fact we can write down

$$
G(z)=P\left(\left(v_{b}, v_{p}\right) \subset D\right)=\iint_{(D)} f\left(v_{b}, v_{p}\right) d v_{b} d v_{p}
$$

In this mathematical expression the parameter $\mathrm{z}$ implicitly enters into integration limits, after differentiation of $G(z)$ on $z$ we will get distribution density for random value $Z$

$$
g(z)=G^{\prime}(z)
$$

Knowing concrete form of function $Z=\phi\left(v_{b}, v_{p}\right)$, we can take $\mathrm{z}$ as integration limits and write down expression for $\mathrm{g}(\mathrm{z})$ in an explicit form. As far as the difference between $v_{p}$ and $v_{b}$ is significant for forecasting of riverside vulnerability, we can consider functional dependence $Z=\phi\left(v_{p}, v_{b}\right)$ in the form of difference between random values

$$
Z=\left(v_{p}-v_{b}\right)
$$


In this case $\mathrm{D}$ area will be represented as semiplane of coordinate plane $\left(v_{b} 0 v_{p}\right)$, which is laid below straight line $v_{p}-v_{b}=Z$. This line cuts coordinate axes into $\mathrm{Z}$ congruent segments (in positive and negative directions)

Random points, which got into this area, satisfy the condition $v_{p}-v_{b}<Z$. In this case distribution function (3) will be as follows:

$$
G(z)=\iint_{(D)} f\left(v_{b}, v_{p}\right) d v_{b} d v_{p}=\int_{+\infty}^{-\infty}\left\{\int_{-\infty}^{z+v_{b}} f\left(v_{b}, v_{p}\right) d v_{p}\right\} d v_{b}
$$

After differentiation of this expression on $\mathrm{z}$ we will get distribution density for random value $Z$

$$
g(z)=\int_{-\infty}^{+\infty} f\left(v_{b}, z+v_{p}\right) d v_{p}
$$

As far as $V_{b}$ and $V_{p}$ are independent random values, $f\left(v_{b}, v_{p}\right)=f_{1}\left(v_{b}\right) \cdot f_{2}\left(v_{p}\right)$.

Thus distribution density (7) for random value $Z$ will be

$$
g(z)=\int_{-\infty}^{+\infty} f_{1}\left(v_{p}\right) f_{2}\left(z+v_{p}\right) d v_{p}
$$

In this case it may be said that takes place composition of two laws, which will be written down as follows:

$$
g=f_{1} * f_{2}
$$

In general cases distribution laws for $f_{1}$ and $f_{2}$ can be of any form, while in our case $V_{b}$ and $V_{p}$ represent random value of normal distribution that is testified by statistical data of long-term observations.

In this case distribution densities $f_{1}$ and $f_{2}$ will be written in following form:

$$
\begin{aligned}
& f_{1}\left(v_{b}\right)=\frac{1}{\delta_{v_{b}} \sqrt{2 \pi}} e^{\frac{\left(v_{b}-m_{v_{b}}\right)^{2}}{2 \delta_{b}^{2}}} \\
& f_{2}\left(v_{p}\right)=\frac{1}{\delta_{v_{b}} \sqrt{2 \pi}} e^{\frac{\left(v_{p}-m_{v_{p}}\right)^{2}}{2 \delta_{p}^{2}}}
\end{aligned}
$$

It is known that composition of two normal distribution laws provides normal distribution law [6,7], at the same time mathematical expectation of new random value is an algebraic sum of start random values, while dispersion is a sum of dispersions.

In our case we will have:

$$
m_{z}=m_{v_{p}}-m_{v_{b}}, \quad \delta_{z}=\sqrt{\delta_{v_{p}}+\delta_{v_{b}}}
$$

Distribution density of random value $\mathrm{Z}$ will be as follows:

$$
g(z)=\frac{1}{\sqrt{\delta_{v_{p}}}+\delta_{v_{b}} \sqrt{2 \pi}} \cdot e^{-\frac{\left[z-\left(m_{v p}-m_{v b}\right)\right]^{2}}{2\left(\delta_{v p}^{2}-\delta_{v b}^{2}\right)}}
$$

while function of its distribution will be:

$$
G(z)=\int_{-\infty}^{z} g(z) d z
$$

As far as there is no risk of riverside washing-off for random value $Z>0$, vulnerability (r) will be assessed according to equation

$$
r=P(Z<0)=G(0)=\int_{-\infty}^{0} g(z) d z
$$

Analysis of obtained formulas shows that increase of $v_{b}$ causes reduction of $m_{z}$, that in its turn increases the area below the curve in the negative area of axis $\mathrm{z}$ and, therefore, assessment of riverside vulnerability at one or another section increases, too.

As far as distribution function $G(z)$ (15) with parameters $\left(m_{z}, \delta_{z}\right)$ can be expressed in the following form by normal distribution function $\Phi^{*}$ of random value with parameters $(0.1)$

$$
G(z)=\Phi^{*}\left(\frac{z-m_{z}}{\delta_{z}}\right)
$$

then the value of riverside vulnerability can be calculated by following formula

$$
r=G(0)=\Phi^{*}\left(-\frac{m_{z}}{\delta_{z}}\right)
$$

\subsection{Defining the Confidence Probability of the Parameters}

Let us consider the following common problem in advance. Say, we have random quantity $\mathrm{X}$, with its distribution law containing unknown parameter $a$. We must find $\widetilde{a}$, which is the relevant evaluation of this parameter and which is gained from $n$ independent experiments, where $\mathrm{X}$ acquires certain values $X_{1}, X_{2} \ldots X_{n}$.. It is clear that this assessment depends on the distribution law of $X$, as well as on the number of experiments ( $\mathrm{n}$ ), and is therefore, a random quantity with the distribution law of unknown value $\widetilde{a}$. In addition, it is a necessary condition that as $n$ increases, the assessment approximates parameter $a$ itself by probability, and its mathematical expectation $M[a]$ equals to $a$. In 
particular, for $m$ mathematical expectation of $\mathrm{X}$ random quantity and $\mathrm{D}$ dispersion, we will have:

$$
\widetilde{m}=\frac{\sum_{i=1}^{n} X_{i}}{n} ; \tilde{D}=\frac{\sum_{i=1}^{n}\left(X_{i}-m\right)^{2}}{n-1}
$$

These values for unknown $\mathrm{m}$ and $\mathrm{D}$ determine the point valuation. Let us note that $\widetilde{m}$ coincides with its statistical analog $m^{*}=\frac{\sum_{i=1}^{n} X_{i}}{n}$ and $\widetilde{D}$ differs from $D^{*}=\frac{\sum_{i=1}^{n}\left(X_{i}-m^{*}\right)}{n}$. However, as $\mathrm{n}$ increases, the difference between $\widetilde{D}$ and $D^{*}$ virtually, abolishes.

First, let us try to determine the confidence interval for the principal parameters of the random quantity, in particular, for mathematical expectation $m$ let us take some high probability $\beta(0.9,0.95 . . \quad \beta(0.9,0.95 \ldots)$ and let us find interval $I_{\beta}$, for which the following condition is true:

$$
P\left(|\widetilde{m}-m|<\varepsilon_{\beta}=\beta\right.
$$

Where $\varepsilon_{\beta}$ is half of width of $I_{\beta}$. Values $\beta$ and $I_{\beta}$ are called confidence probability and confidence interval, respectively. If the distribution law of $\tilde{m}$ had been known, the solution to the problem would have been trivial. In case of unknown distribution law of $\tilde{m}$, a rough method to define the confidence interval is used, which is based on the assumption that the distribution of random quantity $\widetilde{m}$ is normal notwithstanding the kind of law of distribution of initial random quantity $X$. On the other hand, if admitting that the distribution law of random quantity $X$ is normal, then from random quantity $\widetilde{m}$ we can shift to new random quantity $T$ by using the following formula:

$$
T=\sqrt{n} \frac{\tilde{m}-m}{\sqrt{D}}
$$

which obeys to so called Student distribution law with $n-1$ degree of freedom. The distribution density of this law is as follows:

$$
S_{n-1}(t)=\frac{G\left(\frac{n}{2}\right)}{\sqrt{(n-1) \pi} G\left(\frac{n-1}{2}\right)}\left(1+\frac{t^{2}}{n-1}\right)^{-\frac{n}{2}}
$$

Where $G(x)$ is a known gamma-function:

$$
G(x)=\int_{0}^{\infty} u^{x-1} e^{u} d u
$$

By considering (20), expression (19) will be as follows:

$$
P=\left(|T|<\frac{\varepsilon_{\beta}}{\sqrt{\frac{\widetilde{D}}{n}}}\right)=\beta .
$$

By denoting $\frac{\varepsilon_{\beta}}{\sqrt{\frac{\widetilde{D}}{n}}}=t_{\beta}$, we will have:

$$
P\left(|T|<t_{\beta}\right)=\int_{-t_{\beta}}^{t_{\phi}} S_{n-1}(t) d t=\beta
$$

By considering that $S_{n-1}(t)$ is an even function, finally we will have:

$$
2 \int_{0}^{t_{\beta}} S_{n-1}(t) d t=\beta
$$

By solving this equation, the values of $\beta$ given in relation to $t_{\beta}$ and by using the number of $n-1$ degree of freedom, we will find:

$$
\varepsilon_{\beta}=t_{\beta} \sqrt{\frac{\widetilde{D}}{n}}
$$

In reality, there are tables of Student distribution fractiles, where $t_{\beta}$ is defined according to $\beta$ and $(n-1)$.

The confidence interval for mathematical expectation $m$ is defined depending on $\mathcal{E}_{\beta}$, which is symmetrical to $\tilde{m}$ :

$$
I_{\beta}=\left(\tilde{m}-\varepsilon_{\beta} ; \tilde{m}+\varepsilon_{\beta}\right)
$$

The confidence interval of $\mathrm{X}$ random quantity with normal distribution for $D$ dispersion is defined similarly. Assessment of $D$ dispersion in formula (18) is expressed by means of $v$ random quantity, which has $\chi^{2}$ distribution for $n-1$ degree of freedom.

$$
K_{n-1}(\sigma)=\left\{\begin{array}{ccc}
\frac{1}{2^{\frac{n-1}{2}} \Gamma\left(\frac{n-1}{2}\right)} \sigma^{\frac{n-1}{2}-2} e^{-\frac{\sigma}{2}} & \text { when } & \sigma>0 \\
0, & \text { when } & \sigma<0
\end{array}\right\}
$$


As the distribution density of $K_{n-1}(\sigma)$ is not a symmetrical function, the limits of the confidence interval of $v$ random quantity will be selected from the condition of the same probability of the departure from any end of this interval. As the given confidence interval equals to $\beta$, the probability of $v$ not occurring in the confidence interval, equals to $1-\beta$. This condition is met, if the probability of $\mathrm{V}$ random value occurring beyond the right and left ends of the interval equals to $\frac{a}{2}=\frac{1-\beta}{2}$. The tables are drafted for $\chi^{2}$ distribution, according to which, for $p$ probability and $n-1$ number of degree of freedom, the values of $\chi^{2}$ can be found to meet the following condition:

$$
P\left(V>\chi^{2}\right)=p
$$

In our case, for $P_{1}=\frac{\alpha}{2}$ let us find $\chi_{1}^{2}$ and for $P_{2}=1-\frac{\alpha}{2}$ let us find $\chi_{2}^{2}$. Now, we can define $D_{1}$ and $D_{2}$ limits of confidence interval $D$, which corresponds to $\beta-1$ confidence probability:

$$
P\left(D_{1}<D_{2}<D_{3}\right)=\beta
$$

Equation (23) shows that condition $V<\chi_{1}^{2}$ is corresponded by condition $D>D_{1}=\frac{\widetilde{D}(n-1)}{\chi_{1}^{2}}$, while condition $V>\chi_{2}^{2} \quad$ is corresponded by condition $D<D_{2}=\frac{\widetilde{D}(n-1)}{\chi_{1}^{2}}$. Thus, the confidence interval for $D$ dispersion of normally distributed $X$ random quantity $I_{\beta}$, which corresponds to $\beta$ confidence probability and $(n-1)$ number of degree of freedom, will be:

$$
I_{\beta}=\left(\frac{\widetilde{D}(n-1)}{\chi_{1}^{2}} ; \frac{\widetilde{D}(n-1)}{\chi_{2}^{2}}\right)
$$

Now, we can define the confidence interval for $\tau$ value of the river bank, whose point valuation is calculated by the formula:

$$
t=\varphi^{*}\left(-\frac{m_{z}}{\delta_{z}}\right)
$$

Here $Z$ is a random value equaling to the difference between the non-washing (admissible) velocity of the coast
$V_{p}$ and $V_{b}$ bed velocity of the water current $z=\left(V_{p}-V_{b}\right)$, while $\varphi^{*}(x)-$ is the normal parameters of the distribution function (0.1). It is implied that $V_{p}$ and $V_{b}$ are normally distributed values with relevant mathematical expectation and mean square deviation $m_{p}$, $\sigma_{p}$ and $m_{b}, \sigma_{b}$. From here, it is clear that random value $z$ itself is subject to the distribution law with mathematical expectation $m_{z}=m_{p}-m_{b}$ and mean square deviation of:

$$
\delta_{z}=\sqrt{\delta_{p}^{2}-\delta_{b}^{2}}
$$

To calculate the values of $\tau$ by formula (24), point valuations $\tilde{m}_{z}$ and $\widetilde{\delta}_{z}$ are used for $\mathrm{mz}$ mathematical expectation of random quantity $z$ and mean square deviation $\sigma_{z}$, and in line with formula (18) the following values are defined $\widetilde{m}_{p}, \widetilde{D}_{p}$ and $\tilde{m}_{b}, \widetilde{D}_{b}$

$$
\begin{array}{r}
\tilde{m}_{p}=\frac{\sum_{i=1}^{n_{p}} V_{p i}}{n_{p}} ; \tilde{D}_{p}=\frac{\sum_{i=1}^{n_{p}}\left(V_{p_{i}}-\tilde{m}_{p}\right)^{2}}{n_{p}-1} \\
\tilde{m}_{b}=\frac{\sum_{i=1}^{n_{b}} V_{b i}}{n_{b}} ; \tilde{D}_{b}=\frac{\sum_{i=1}^{n_{b}}\left(V_{b i}-\tilde{m}_{b}\right)^{2}}{n_{b}-1}
\end{array}
$$

Where $n_{p}$ and $n_{p}$ are the numbers of the observation data of random quantities $V_{p}$ and $V_{p}$, respectively.

By considering $\tilde{m}_{z}$ and $\widetilde{\delta}_{z}$ randomness, to strictly define the limits of $Z$ confidence interval, we must consider a new random quantity $V=-\frac{\widetilde{m}_{z}}{\widetilde{\delta}_{z}}$ and its distribution law must be identified.

After identifying the limits of $m_{y}$ confidence interval of the mathematical expectation of value $Y$, we can fix the limits of $z$ confidence interval, $z_{1}$ and $z_{2}$, from formula (24):

$$
z_{1}=\varphi^{*}\left(y_{1}\right) \text { and } z_{2}=\varphi^{*}\left(y_{2}\right)
$$

Identification of the distribution law of random quantity $y$ entails certain difficulties leading to an unjustified complication of the problem [8,9]. However, if considering the slight dependence of $z$ on the form of distribution density of random quantity $z$, i.e. $\sigma_{z}$ and essential dependence on the location of the dissipation center, on axis 
$z$ (i.e. on $m_{z}$ ), then, in formula (24), instead of parameter $\delta_{z}$, we may use its point evaluation $\widetilde{\delta}_{z}$, which by the first receiver may be considered a permanent value. Then, confidence interval $(\tau)$ of vulnerability is defined with the confidence interval of $m_{z}$, which will be taken symmetrically from its point evaluation $\tilde{m}_{z}=\widetilde{m}_{p}-\tilde{m}_{b}$, for the same probability $(\beta)$ for $m_{p}$ and $m_{b}$, the confidence interval can be defined by the same confidence probability $\left(I_{\beta}\right)$ for $m_{x}$. We can note that as the number of degree of freedom $(n-1)$ is reduced, the degree of indefiniteness to asses the mathematical expectation of random quantity $m$ increases, i.e. the confidence interval increases for the same confidence probability. Therefore, the number of degree of freedom for $m_{x}$ cannot be more than the minimum values of the numbers of degree of freedom for $m_{p}$ and $m_{b}$ [10].

Thus, if taking $\beta$ as confidence probability and $K=\min \left(n_{p}, n_{b}\right)-1$ as a number of degree of freedom for $m_{z}$, from the Student distribution equation we can identify $t_{\beta}$, then from formula (22) we can identify $\varepsilon_{\beta}$ and consequently, the lower and upper limits of the confidence interval of $m_{1}$ and $m_{2}$ (22):

$$
\varepsilon_{\beta}=t_{\beta} \sqrt{\frac{\widetilde{D}_{z}}{K+1}} ; m_{1}=\tilde{m}-\varepsilon_{\beta} ; \quad m_{2}=\tilde{m}+\varepsilon_{\beta}
$$

By inserting the values of $m_{1}$ and $m_{2}$ in formula (24), we will gain the confidence interval for the lower and upper limits for $\tau_{1}$ and $\tau_{2}$ vulnerability, with its confidence interval of $\beta$ :

$$
z_{1}=\Phi^{*}\left(-\frac{m_{1}}{\sqrt{\widetilde{D}_{p}+\widetilde{D}_{b}}}\right) ; z_{2}=\Phi^{*}\left(-\frac{m_{2}}{\sqrt{\widetilde{D}_{p}+\widetilde{D}_{b}}}\right)
$$

Thus, by $\beta$ probability, the value of vulnerability of the river bank will not go beyond the interval:

$$
I_{\beta}=\left[\Phi^{*}\left(-\frac{\tilde{m}-\varepsilon_{\beta}}{\sqrt{\widetilde{D}_{p}+\widetilde{D}_{b}}}\right) ; \Phi^{*}\left(-\frac{\tilde{m}+\varepsilon_{\beta}}{\sqrt{\widetilde{D}_{p}+\widetilde{D}_{b}}}\right)\right]
$$

\section{Results}

Assume, we have the observation data subject to the normal distribution law. For the admissible river current velocity $n_{p}=15$, mathematical expectation $m_{p}=3$ $\mathrm{m} / \mathrm{sec}$, mean square deviation $\sigma_{p}=0.9$; for bed velocity, $n_{b}=20 ; m_{b}=4 \mathrm{~m} / \mathrm{sec}$ and $\sigma_{b}=1.3$. We must identify the confidence interval of the bank vulnerability.

To imitate the given random quantities, let us use the table of values of $\bar{\xi}$ random quantity of Gauss, with its mathematical expectation of $a=0$ and its mean square deviation of $\sigma=1$. It is easy to prove that random quantity $\xi^{\prime}=a+\sigma_{\xi} \xi^{\prime}$ (25), which will be subject to the normal distribution law, with mathematical expectation of $m\left[\xi^{\prime}\right]=\alpha$ and dispersion sion $D\left[\xi^{\prime}\right]=\sigma^{2}$.

By subsequent use of formula (25) and Gauss table of values of $\bar{\xi}$ random quantity, we will calculate the values of $V_{p}$ and $V_{b}$ :

$$
\begin{aligned}
& V_{p 1}=3+0.9 \cdot 0.2005 \approx 3.18 ; \quad V_{p 2}=3+0.9 \cdot 0.1922 \approx 4.07 ; \ldots \\
& V_{b 1}=4+1.3 \cdot 0.2005 \approx 4.26 ; \ldots
\end{aligned}
$$

\begin{tabular}{|c|c|c|c|c|c|c|c|c|c|c|c|c|c|c|c|}
\hline $\bar{i}$ & 1 & 2 & 3 & 4 & 5 & 6 & 7 & 8 & 9 & 10 & 11 & 12 & 13 & 14 & 15 \\
\hline$V_{p 1}$ & 3.80 & 4.07 & 2.99 & 3.03 & 3.94 & 1.37 & 4.06 & 3.00 & 4.04 & 2.40 & 1.57 & 3.52 & 4.69 & 3.66 & 2.75 \\
\hline
\end{tabular}

Let us put the calculated values in the table 1 and table 2:

Table 1. Calculate the values of $V_{p}$.

Table 2. Calculate the values of $V_{b}$.

\begin{tabular}{lllllllllllllllllllll}
\hline$i$ & 1 & 2 & 3 & 4 & 5 & 6 & 7 & 8 & 9 & 10 & 11 & 12 & 13 & 14 & 15 & 16 & 17 & 18 & 19 & 20 \\
$V_{b 1}$ & 4.26 & 5.55 & 3.93 & 4.04 & 5.35 & 1.64 & 5.50 & 4.00 & 5.53 & 3.13 & 1.93 & 4.76 & 6.45 & 4.96 & 3.64 & 5.40 & 4.70 & 2.80 & 4.12 & 5.92 \\
\hline
\end{tabular}

Let us calculate the relevant values from formula (25):

$$
\begin{aligned}
& \widetilde{m}_{p=48.27: 15=3.22 ;} \widetilde{D}_{p=12.2407: 14=0.874 ;} \quad \widetilde{\delta}_{p=0.935} \\
& \widetilde{m}_{b}=87.78: 20=4.39 ; \quad \widetilde{D}_{b}=31.9652: 19=1.682 ; \quad \widetilde{\delta}_{b}=1.297
\end{aligned}
$$

For random quantity $z=V_{p}-V_{b}$ we will have:

$$
\begin{aligned}
& \widetilde{m}_{z}=\widetilde{m}_{p}-\widetilde{m}_{b}=3.22-4.97=-1.17 \\
& \widetilde{D}_{z}=\vec{D}_{p}-\widetilde{D}_{b}=0.874+1.682=2.56
\end{aligned}
$$




$$
\widetilde{\delta}_{z}=\sqrt{\widetilde{D}_{z}}=\sqrt{2.56}=1.6
$$

From formula (24) and table of the values of distribution of normal function, we identify the $z$-point evaluation of the vulnerability of the river bank:

$$
z=\Phi^{*}\left(-\frac{\widetilde{m}_{z}}{\widetilde{\delta}_{z}}\right)=\Phi^{*}\left(\frac{-1.17}{1.6}\right)=\Phi^{*}(0.73) \approx 0.77
$$

Now, let us determine confidence interval for $\mathrm{mz}$, with confidence probability of $\beta=0,95$. As $\min \left(n_{p}, n_{b}\right)=$ $\min (15,20)=15$, then the degree of freedom will equal to $K$ $=15-1=14$. From the table of distribution of the given Student fractiles $\beta$ and $K$ we find $t_{0.95}=2.14$.

From here, by using formula (26) we define:

$$
\varepsilon_{0.95}=t_{0.95} \sqrt{\frac{\widetilde{D}_{z}}{K+1}}=2.14 \sqrt{\frac{2.56}{15}} \approx 0.88
$$

Consequently, the lower and upper limits of the confidence interval for values $m_{1}$ and $m_{2}$ will equal to:

$$
\begin{aligned}
& m_{1}=\tilde{m}_{z}-\varepsilon_{0.95}=-1.17-0.88=-2.05 \\
& m_{2}=\tilde{m}_{z}+\varepsilon_{0.95}=-1.17+0.88=-0.29
\end{aligned}
$$

Consequently, from table of the normal function distribution and formula (27) we identify $z_{1}$ and $z_{2}$ limits of the confidence interval for the vulnerability of a river bank:

$$
\begin{aligned}
& z_{1}=\Phi^{*}\left(-\frac{\widetilde{m}_{z}}{\widetilde{\delta}_{z}}\right)=\Phi^{*}\left(\frac{2.05}{1.6}\right)=\Phi^{*}(1.28)=0.9 \\
& z_{1}=\Phi^{*}\left(-\frac{\widetilde{m}_{z}}{\widetilde{\delta}_{z}}\right)=\Phi^{*}\left(\frac{2.05}{1.6}\right)=\Phi^{*}(1.28)=0.9
\end{aligned}
$$

Finally, the vulnerability of a river bank with 0.95 probability will be within the interval of $I_{0.95}=(0.57 ; 0.9)$ with point evaluation $z=0.77$.

\section{Conclusions}

Among natural processes freshets represent such phenomenon, which was, is and will be the reason of biggest damage for population of any country. At present there is no methodology, which makes possible forecasting of risks related to river bed coastal degradation with permissible accuracy in order to timely take measures for getting rid of expected damage. That's why a mentioned phenomenon will remain so far the research subject for appropriate field of science.

Mechanism of riverside destruction by water flow is considered in the presented work as random process, which is depended both on influence of flow speed and riverside resistance. As the indicator of this process against such influence is taken riverside characteristic - vulnerability, for determination of which is used a well-known model of the theory of reliability, called "load-strength" model. Proceeding from this fact a result obtained via theoretical formalization in the form of represented formula has to be considered as rough approximation and it can't claim the high accuracy.

Within the frameworks of initiated researches is scheduled a follow-up of works with the end of removal of current disadvantages, namely determination of limits for approximate value of vulnerability with predefined accuracy and taking into account time factor in the modeling process that will be a step forward in relation to current reality.

\section{References}

[1] P. Shatberashvili, G. Dokhnadze, D. Kereselidze."Floods and freshets in western Georgia, freshet-preventive and erosion-preventive measures". In collection: Engineering methods of forecasting and fight against soil erosion, Tbilisi, 1977, pp. 133-142.

[2] G. Dokhnadze, Z. Charbadze, D.Lortkipanidze, L.Tsanava. "Freshets on Rioni River and establishment of probable values of maximal expenses", Georgian Academy of Sciences, Institute of water economy, collection of scientific works.

[3] B. V. Gnedenko, Yu. K. Belyaev, A. L. Solovyov, "Mathematical methods in the theory of reliability". Moscow, "Nauka", 1965, p. 524.

[4] Sotskov B. S."Basics of the theory of reliability of elements and devices of automatics and computing techniques", Moscow, "Vysshayashkola", 1970, p. 270.

[5] Ts. E. Mirtskhulava, "Hazards and risks at water and other systems" (in 2 volumes), Tbilisi, "Nauka”, 2003, p. 537.

[6] E. S. Wentzel."Theory of probability", Moscow, "Vysshayashkola", 1969, p. 576.

[7] B. V. Gnedenko, "The course of theory of probability", Moscow, Publishing house "Phizmatgiz", 1961, p. 406.

[8] D. Kereselidze, K. Bilashvili,V. Trapaidze. "Hydrological zoning of the territory of Georgia and estimation of water resources on the background of the climatic change"Proccedings 12-th International Multidisciplinary Scientific Geo-Conference \& EXPO - SGEM 2012, vol III, pp-729-732.

[9] D. Kereselidze, L. Matchavariani, V. Trapaidze, et al. Evaluation and Management of the Risk of Flooding River Bank. Engineering Geology for Society and Territory. Vol.3: River Basin, Reservoir Sedimentation and Water Resources. SPRINGIER International Publishing Switzerland, pp. 463-469, 2014

[10] D. Kereselidze, V. Trapaidze, G.Bregvadze. "The methods for determination of hydrological specifications". "Universal" 2009, p.120. 